\title{
Shape Interactions in Macaque Inferior Temporal Neurons
}

\author{
MARCUS MISSAL, RUFIN VOGELS, CHAO-YI LI, AND GUY A. ORBAN \\ Laboratorium voor Neuro- en Psychofysiologie, Faculteit Geneeskunde, Katholieke Universiteit Leuven, \\ B-3000 Leuven, Belgium
}

\begin{abstract}
Missal, Marcus, Rufin Vogels, Chao-Yi Li, and Guy A. Orban. Shape interactions in macaque inferior temporal neurons. J. Neurophysiol. 82: 131-142, 1999. Missal et al. observed that the responses of inferior temporal (IT) neurons to a shape were reduced markedly when this shape partially overlapped a larger second shape, suggesting that shape interactions determine IT responses. In the present study, we compared the responses of IT neurons with combinations of two shapes which did or did not overlap and studied the effect of the relative and absolute positions of the two shapes. In a first test, a preferred shape (figure) was presented at the fixation point while a second, nonpreferred, shape was displayed either in the background of the figure (overlap) or at one of four peripheral positions (nonoverlap). Controls consisted of presentations of either shape in isolation at each of the five positions. The stimuli were presented during a fixation task. The responses to these combinations of two shapes were, on average, reduced compared with those elicited by the preferred shape presented in isolation. This suppression occurred whether or not the two shapes overlapped, but the degree of suppression in the overlap and nonoverlap conditions did not correlate. These interactions were stronger when the interacting stimulus was located in the contralateral compared with the ipsilateral hemifield. The position of the interacting stimulus within a hemifield significantly affected the suppression associated with combined shapes in some neurons. The strength of the interactions of the two nonoverlapping shapes depended on the shape of the interacting stimulus in half of the neurons. In a second test, the preferred shape and interacting stimulus could appear either at the fixation point or at one eccentric position. Here we found that the suppression was, on average, strongest when the interacting stimulus, rather than the preferred shape, was presented at the fixation position. Also, in $40 \%$ of the neurons, the response reduction was similar in overlap and nonoverlap conditions if effects of stimulus position were taken into account. In both tests, we also measured the responses to combinations of a nonpreferred shape and the interacting stimulus and showed that the response to a combination of two nonpreferred shapes was, in general, smaller than the response to a combination of the preferred and nonpreferred shape. Overall the results indicate that stimulus interactions in the receptive fields of IT neurons can be position and shape selective; this can contribute to the coding for the relationships between object parts.
\end{abstract}

I N T R O D U C T I O N

Neurons in the inferior temporal (IT) cortex are selective for object attributes such as shape, color, and texture (Desimone et al. 1984; Gross et al. 1972; Komatsu et al. 1992; Tanaka et al. 1991), and lesions of this part of the temporal cortex produce deficits in object recognition (for review, see Dean 1976). These and other findings suggest that IT plays an important role in the complex process of object recognition (see review of Logothetis and Sheinberg 1996).

The costs of publication of this article were defrayed in part by the payment of page charges. The article must therefore be hereby marked "advertisement" in accordance with 18 U.S.C. Section 1734 solely to indicate this fact.
In a previous study (Missal et al. 1997), we measured the responses of IT neurons to two overlapping shapes, a preferred shape overlapping a larger nonpreferred shape, the two of which were perceptually segmentable because they differed in color or texture. We observed that the response to this combination usually was reduced sharply compared with the response to the preferred shape, presented in isolation. These shape interactions, which depended on the shape of the background stimulus, potentially could enhance the stimulus selectivity of the neuron, making it sensitive to combinations of shapes.

Other studies (Miller et al. 1993; Rolls and Tovee 1995; Sato 1988) have reported smaller responses when IT neurons are stimulated by two nonoverlapping stimuli compared with a single stimulus (but see Sato 1989, 1995). The first aim of the present study is to compare the degree of suppression for overlapping and nonoverlapping shapes. In the case of overlapping shapes, the decrease in response may be due in part to factors linked to the overlap per se, such as a weak sensitivity of the IT neurons to the segmentation or overlapping shapes being treated as a single structural unit. If so, one might expect diminished or no suppression for nonoverlapping compared with overlapping shapes because, in the nonoverlap condition, the two shapes are segregated. On the other hand, neurons may show similar suppression for overlapping and nonoverlapping shapes, reflecting similar shape interactions in the two conditions.

A second aim was to determine the sensitivity of IT neurons to the spatial configuration of two shapes. Indeed the strength of the stimulus interactions may depend on the absolute (receptive field) position and relative position of the two shapes. Sato (1988) found less suppression from ipsilateral compared with contralaterally positioned stimuli during active discrimination of sequentially presented simple stimuli, but Rolls and Tovee (1995), testing face cells in a fixation task, reported that the hemifield had no effect on the suppression. Given these results and because the effects of altering the absolute position of an interacting shape have not been studied systematically where both shapes are presented simultaneously during fixation, we measured the effect of the angular and radial position of the interacting shape on the responses to a centrally presented shape. The central and interacting shape consisted of a preferred and nonpreferred shape, respectively. In this first experiment, we also measured responses to the single preferred shape at the different positions so we could determine whether any position effects observed with the interacting stimulus merely reflected the receptive field sensitivity profile of the neuron. In addition to varying the position of the interacting stimulus, we also varied its shape in a limited number of neurons. If the position and/or shape of the nonoverlapping 
interacting stimulus show an effect, this would provide further evidence for a coding of the relationships between shapes in IT.

In the first experiment, a difference in the responses to the overlap and nonoverlap conditions could be due to either the different absolute positions of the interacting shape or the overlap factor (relative position). In a second experiment, we disentangled absolute and relative position by manipulating the position of the two shapes independently.

Unlike previous studies, the responses to the combination of the same interacting shape and either a preferred shape or a nonpreferred shape were measured in both experiments. One can identify both of the latter shapes, despite the presence of the interacting shape (for behavioral evidence in the monkey in the case of overlapping shapes, see Missal et al. 1997). Thus the third aim was to determine to what extent the shape interactions alter the shape preference of an IT neuron, i.e., whether neurons still respond more strongly to a preferred shape presented together with another shape than to a combination of two nonpreferred shapes.

Part of this work has been presented earlier in a preliminary form (Missal et al. 1996).

\section{METHODS}

\section{Surgery}

Two juvenile male macaque monkeys (Macaca mulatta) were used as subjects ( $T$ and $J$ ). These subjects also were used in a previous set of experiments (Missal et al. 1997). During experiments, monkeys were water deprived but received dry food ad libitum supplemented by fruits when necessary.

Both monkeys had a scleral search coil implanted in one eye (Judge et al. 1980) and a stainless steel peg cemented to the skull for head fixation. Single cells were recorded through a recording chamber placed dorsolaterally to IT. All surgical procedures were performed under deep anesthesia and aseptic conditions. The procedures were conformed to the guidelines established by the National Institutes of Health for the care and use of laboratory animals.

\section{Apparatus}

The apparatus is identical to the one of Missal et al. (1997). Briefly, eye movements were recorded with the search coil technique and single-cell recordings were made with tungsten electrodes (World Precision Instruments, impedance 1.5-2.0 M $\Omega$ ) lowered into a guiding tube. Single cells were isolated using a time-amplitude window discriminator (Bak Electronics, model DIS-I). The times of spike occurrence, stimulus, and behavioral events were displayed and stored by a PC. Stimuli were generated by a Silicon Graphics Indy computer and displayed on a Sony Trinitron screen $(1,024 \times 1,024$ pixels; width: $39^{\circ}$, height: $29.5^{\circ}$ ) at $57 \mathrm{~cm}$ from the monkey's eyes.

\section{Stimuli and behavioral paradigm}

We used a set of 30 computer-generated polygonal shapes, the same as those used by Missal et al. (1997). The median of the largest diameter (distance between the 2 extreme apices) was $3.0^{\circ}$ (quartiles, 2.7-3.3). All the shapes except one (a circle) were asymmetrical. These stimuli ranged from simple (circle) to fairly complex geometric shapes (Fig. 1) (see also Missal et al. 1997 for examples of actual shapes). Shapes were displayed on a uniform black screen (luminance $0.02 \mathrm{~cd} / \mathrm{m}^{2}$ ) as a uniform surface of a given color and luminance. Luminance of the shapes depended on their color: $71.9 \mathrm{~cd} / \mathrm{m}^{2}$ for white, $18.8 \mathrm{~cd} / \mathrm{m}^{2}$ for red, $49.7 \mathrm{~cd} / \mathrm{m}^{2}$ for green, and $5.4 \mathrm{~cd} / \mathrm{m}^{2}$ for

\section{A Single shape \\ Ipsi Up (III) Contra Up (II)}

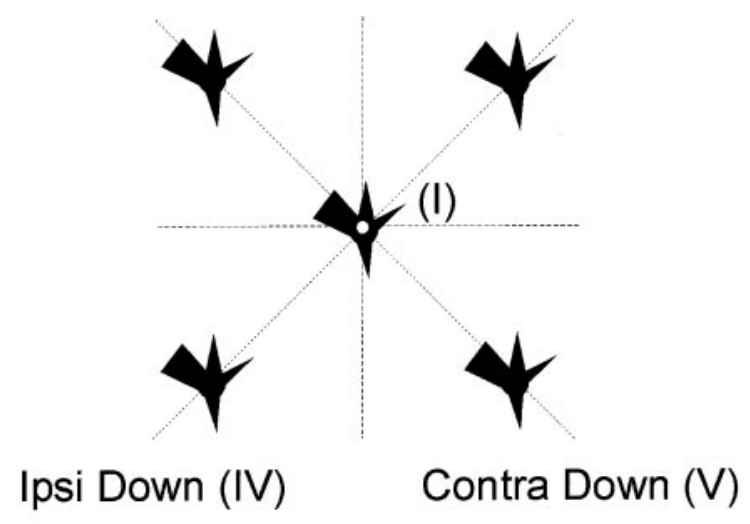

B Shape combination

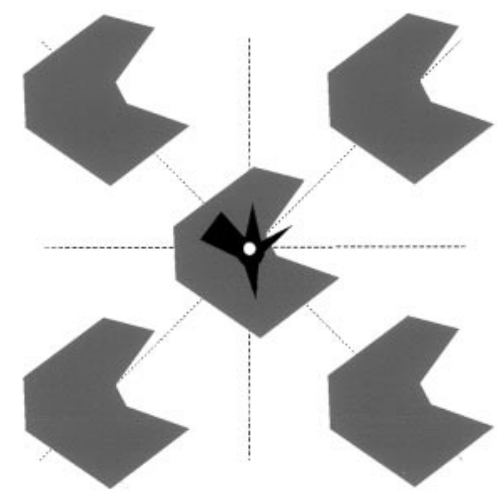

FIG. 1. Position test. A: conditions with a single shape. Preferred shape (figure) was shown at the displays' center (position I) or at 1 of 4 eccentric positions $(I I-V)$. B: conditions with 2 combined shapes. Preferred figure was positioned at the display's center and the larger interacting stimulus either at the center (overlap condition) or at 1 of the 4 eccentric positions (nonoverlap). In all conditions, a fixation target was presented in the center of the display during stimulus presentation as indicated by the small white dot. Ipsi, ipsilateral; Contra, contralateral visual field.

blue. Centering of the shapes was achieved by aligning their center of mass.

A trial started with the onset of a fixation target (22-min arc diameter) in the center of the display. After fixation had been held on the target for $700 \mathrm{~ms}$, the stimulus was presented for $300 \mathrm{~ms}$. If the monkey maintained its gaze within the square $(1.5 \times 1.5 \mathrm{deg})$ fixation window during the stimulus presentation, a juice reward was delivered. The stimulus consisted either of a single shape or of two shapes presented simultaneously. Inter-trial interval was $1,000 \mathrm{~ms}$.

\section{Initial testing}

Each neuron was tested first with the set of 30 shapes, presented in isolation at the display's center in each of four colors (white, red, green, and blue). In this and subsequent tests, stimulus conditions were interleaved. If a cell responded to at least one of the shapes in any color, it was tested further. For each cell, three different shapes were selected: one shape evoking a strong response, defined as the 
preferred figure, and two other shapes evoking no or only a modest response, one to be used as the nonpreferred figure and the other as the interacting stimulus. The preferred and nonpreferred figures were presented either alone or in combination with the interacting stimulus in a different color (see Figs. 1 and 2). The interacting stimulus was 1.6 times the size of the figure so that its border was still visible when figure and interacting stimulus overlapped. One or the two tests described in the following text then were performed.

\section{Position test}

In this test, we measured the effect of the position of an interacting stimulus on the response to two shapes, the preferred or nonpreferred figure, which were positioned at the display's center. This test consisted of 21 conditions, 10 of which are illustrated in Fig. 1. In the first five conditions, the preferred figure was presented in the display's center (position I) or at each of four different eccentric positions
1

A

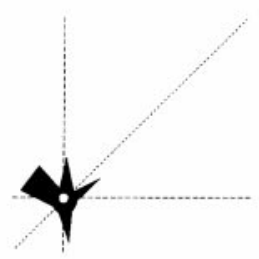

3

B

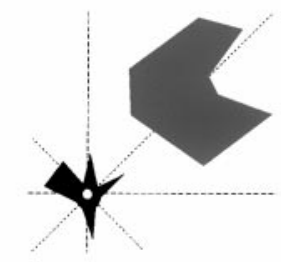

5

C

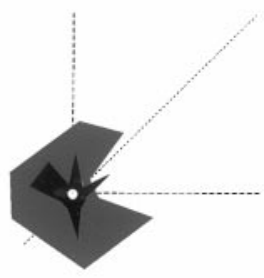

7

D

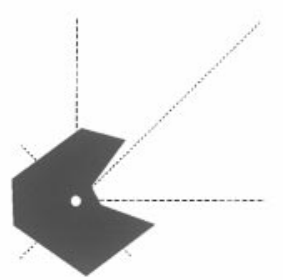

2

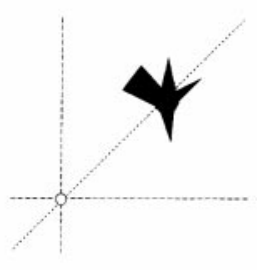

4

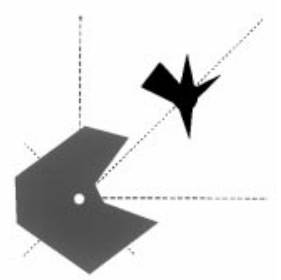

6

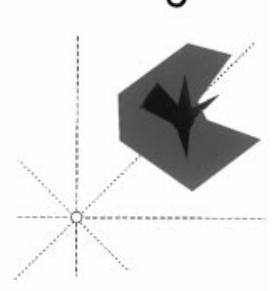

8

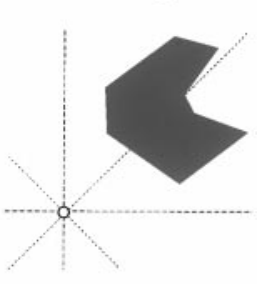

FIG. 2. Absolute and relative position test. A: single, preferred figure positioned either in the display's center (1) or at the contralateral upper position (2). $B: 2$ nonoverlapping shapes in the RF. Figure was positioned either in the center, with the interacting stimulus in the periphery (3), or in the periphery with the interacting stimulus in the center (4). $C$ : figure and interacting stimulus overlapping in the center (5) or in the periphery (6). $D$ : single, interacting stimulus positioned in the center (7) or in the periphery (8). (positions II-V; see Fig. 1A). These conditions allowed us to measure the responses of the neuron as a function of the position of the preferred shape. In the next five conditions, the interacting stimulus was presented in isolation in the center $(I)$ or at the same eccentric positions ( $I I-V$; not shown) to determine the response to this second stimulus alone. In the following five conditions, the preferred figure was always displayed at the center position with the interacting stimulus either behind it so that the two overlapped (Fig. 1B, position $I$ ) or in one of the four eccentric positions (Fig. $1 B$, positions $I I-V$ ), where there was no overlap. In the next five conditions the nonpreferred figure was displayed in the center with the interacting stimulus either behind it or shifted to the one of the four eccentric positions. Last, we measured the response to the nonpreferred figure presented in isolation at the display's center.

The eccentricity of the stimulus was adapted (range: $1.8-10.8^{\circ}$ ) to the extent of the receptive field (RF). To that end, the preferred figure was displayed at decreasing eccentricities until a reliable response was evoked in at least one of the four eccentric positions. Thirty-eight of the 43 neurons tested responded significantly at both contralateral positions. The same eccentricity was maintained for all conditions within a given position test. The eccentric positions were located along the two diagonals through the display's center: two positions were in the visual field contralateral to the recording site and two were ipsilateral (see Fig. 1).

\section{Absolute and relative position test}

The interacting stimulus and the figure could appear either in the display's center or in a single eccentric position that was where the response to the preferred figure was strongest (usually in the upper contralateral part of the receptive field). In fact, all cells responded significantly at the eccentric position in this second test. Both stimuli were presented simultaneously, either at the same position (overlap conditions) or in the two different positions (nonoverlap conditions). Figure 2 depicts 8 of the 14 conditions of this test. In the first two conditions, the preferred figure was presented in the center or eccentric position (Fig. $2 A$, conditions 1 and 2). In the next two, the preferred figure and the interacting stimulus were presented in different positions (Fig. 2B, conditions 3 and 4 ). In the fifth and sixth conditions, the preferred figure and interacting stimulus overlapped (Fig. 2C, conditions 5 and 6 ). Notice that conditions 3 and 5 were included in the position test but not conditions 4 and 6 . In the next two conditions, the interacting stimulus alone was displayed in the center or in the periphery (Fig. 2D, conditions 7 and 8). The final six conditions consisted of the same stimulus configurations as conditions $1-6$, except that a nonpreferred shape was used as the figure.

In both the position and the absolute and relative position tests, $\geq 10$ unaborted trials were recorded for each condition.

\section{Data analysis}

Off-line spikes were counted trial-wise within a 300-ms window that started $50 \mathrm{~ms}$ after stimulus onset. Net responses were calculated by trial-wise subtraction of the activity during an interval of the same duration as the stimulus time window but just preceding stimulus onset.

Cells were included in the analysis if there was a statistically significant shape-selective response to the single stimuli of the position test. This was tested by ANOVA (Kirk 1968). The eccentric positions were considered part of the receptive field of a neuron if the preferred figure presented alone at that eccentric position evoked a significant response compared with the spontaneous activity (Wilcoxon matched pairs test; $P<0.05$ ). The effect of the position of the preferred figure or of the combination of figure and interacting stimulus were tested for each cell individually by ANOVAs and by post hoc comparison tests using the net responses in individual trials. Correlation between responses in the different conditions or popula- 
tion analyses were performed on normalized responses: the net response in a particular condition was divided by the net response to the preferred figure presented in isolation at the display's center.

Selectivity indices (SI) were computed for the center and each of the four eccentric positions of the interacting stimulus as follows. For figures in isolation, the SI was obtained by subtracting the average net response to the nonpreferred figure in the center from the average net response to the preferred figure in the center and then dividing this difference by the response to the preferred figure. In the conditions with an additional presence of an interacting stimulus, the SI was computed by subtracting the average net response to the combination of nonpreferred figure and interacting stimulus from the combination of preferred figure and interacting stimulus and dividing this difference by the response to the preferred figure alone in the center. This is equivalent to subtracting the responses normalized with respect to the response to the figure alone presented at the display's center.

\section{RES U L T S}

We recorded from 57 shape selective cells in the IT cortex of two monkeys ( 15 cells in monkey $T$ and 42 cells in monkey $J ; 43$ and 17 neurons in the first and second test respectively). These two monkeys also were used in a previously published set of experiments (Missal et al. 1997). Therefore the position of the recording sites will be described only briefly (see Fig. 2 in Missal et al. 1997). Histological analysis of the brain of both monkeys showed that all the recording sites were located in the anterior part of IT, area TE, and excluded area TEO. Neurons were recorded in the lower bank of the superior temporal sulcus and in the convexity of the inferior temporal gyrus.

\section{Position test}

COMPARISON OVERLAPPING VERSUS NONOVERLAPPING SHAPES. All neurons responded when the preferred shape was presented in isolation at the fixation position (mean response strength \pm $\mathrm{SD}=31 \pm 18 \mathrm{spikes} / \mathrm{s} ; n=43$ ). All neurons were tested with the preferred figure at $5.5^{\circ}$ eccentricity (most neurons also were tested at other eccentricities). A reliable response was evoked by the single preferred figure in at least one of the four positions at this $5.5^{\circ}$ eccentricity in $72 \%$ of the neurons. On average, responses were similar in the center and at $5.5^{\circ}$ eccentricity in the contralateral field [mean response strength: 27 spikes/s (mean of upper and lower positions)] but were lower in the ipsilateral field (mean response strength $=13$ spikes/s).

Adding a second shape either in the background or peripheral to the preferred figure usually reduced the response of the neuron. On average, the response was most strongly reduced (mean $\pm \mathrm{SD}: 38 \pm 42 \%$ reduction; $n=43$ ) when the preferred figure and interacting stimulus overlapped. With the preferred figure at the center and the interacting stimulus in the lower or the upper contralateral field (nonoverlapping conditions) the average response was reduced by $31 \pm 30 \%$ or $20 \pm 46 \%$, respectively. For the lower and upper ipsilateral positions of the interacting stimulus, the response reduction was $16 \pm 35 \%$ and $9 \% \pm 28 \%$, respectively. Thus both overlapping and nonoverlapping conditions produced response suppression.

To present the effect on the interacting stimulus, the neurons were categorized in four groups using a criterion of $50 \%$ response reduction in overlap and/or nonoverlap conditions. Nine of the 43 neurons showed response decreases of $\geq 50 \%$ when the interacting stimulus was placed in the center and in one or more eccentric positions. An example of such a neuron is shown in Fig. 3. Figure $3 A$ shows the responses of the cell to the preferred and nonpreferred figures presented alone. The neuron responded to the preferred figure at each of the tested positions. Figure $3 B$ shows that the presentation of the interacting stimulus had a significant effect on the response to the preferred figure [ANOVA; $F(5,54)=27.7 ; P<0.001$ ]. A significant response decrease (post hoc comparisons of means, LSD test; $P<0.01$ ) was observed both when the interacting stimulus was present in the background or in either of the two contralateral positions (Fig. 3B, dark bars). Decreases were greatest at the center position, and the interacting stimulus had no effect on the response to the preferred figure when placed in the ipsilateral part of the receptive field. It should be noted that the responses to the interacting stimulus in isolation (shaded bars) were weak or absent at all five positions.

Ten other neurons gave reduced responses of $\geq 50 \%$ when the interacting stimulus was presented at an eccentric position but not when the two shapes overlapped. Such a neuron is illustrated in Fig. 4. It responded to the preferred figure in the center and in the two contralateral positions (Fig. 4A; open bars). A significant decrease in response was observed when the interacting stimulus was displayed contralaterally (LSD post hoc test; $P<0.001)$. No significant decrease was observed with the interacting stimulus in the center or ipsilateral region of the receptive field (Fig. 4B, black bars). Notice that the preferred figure on its own evoked a clear response in the center position. In a third group $(n=12)$ of neurons, the interacting stimulus reduced the response by $\geq 50 \%$ only when the two shapes overlapped. The remaining 12 cells showed less than a $50 \%$ reduction in any position.

These four groups of neurons are not really distinct but serve to illustrate the different types of correlation between the degree of response suppression in the overlap and nonoverlap conditions. These results indicate that interactions induced by shapes in the center and periphery are not necessarily related. This was confirmed by correlating the normalized responses to the preferred figure with the interacting stimulus in the center (shape overlap) and those with the interacting stimulus in eccentric positions (nonoverlapping shapes). This correlation analysis was restricted to the contralateral positions in which the interacting stimulus was the most effective. Neither correlation was significant: $r=0.19(n=43$; ns) for the upper position and $r=0.22$ ( $n=43$; ns) for the lower position. Overall, these data imply that testing either the overlap (Missal et al. 1997) or nonoverlap conditions (Miller et al. 1993) will underestimate the prevalence of shape interactions in IT. It should be noted that the response to the combination of the two shapes increased by $\geq 50 \%$ in only 3 of the 43 neurons tested, indicating the predominantly suppressive nature of the shape interactions.

EFFECT OF ABSOLUTE POSITION OF THE NONOVERLAPPING INTERACTING STIMULUS. On average, positions in the contralateral visual field were more effective in reducing the response to the central preferred figure than those in the ipsilateral visual field (e.g., Figs. 3 and 4). To test the influence of the hemifield in which the interacting stimulus was displayed, we performed an ANOVA for all cells after pooling the normalized responses to the preferred figure plus the interacting stimulus within each hemifield. This test confirmed that the 
A
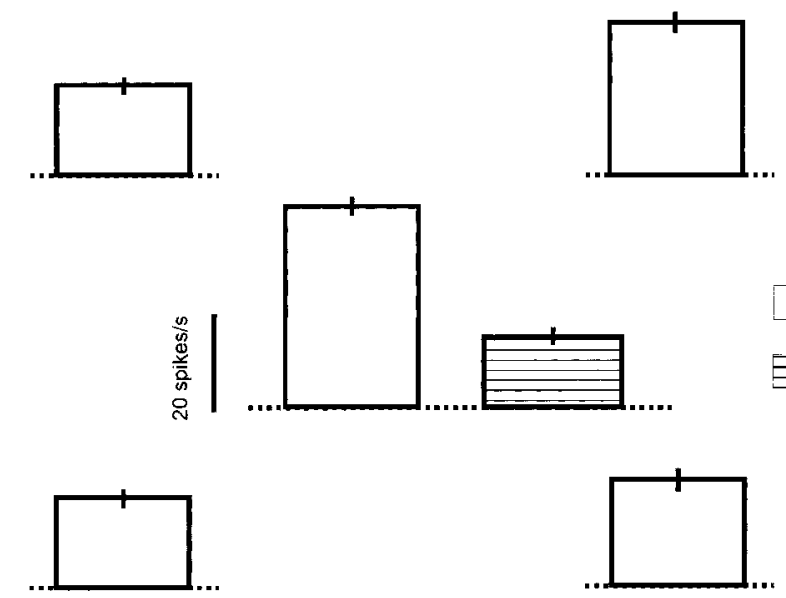

B
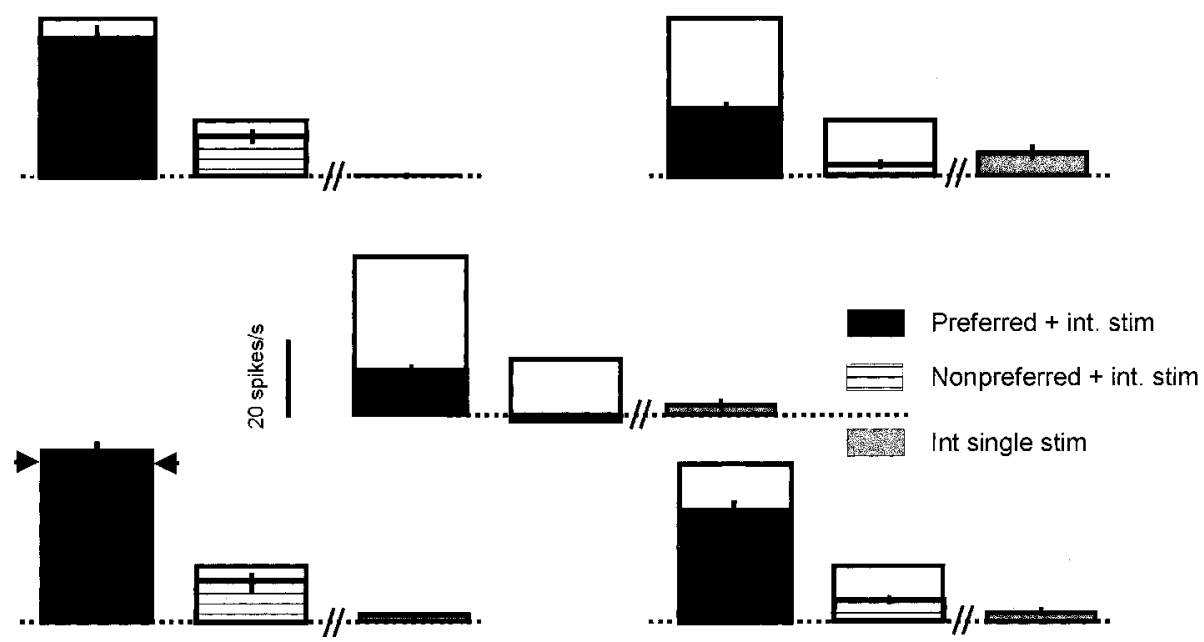

FIG. 3. Responses of an inferior temporal (IT) neuron showing response suppression with the combined shapes, regardless of whether there was overlap between the 2 shapes. A: mean response to single figures. Open bars, preferred figure at each of the 5 positions; hatched bar, nonpreferred figure at the center. Position of the bar represents the visual field position of the figure (see Fig. $1 A$ ), e.g., top right bar indicates the response to the preferred shape positioned in the upper contralateral visual field. $B$ : mean response to combined stimuli and to interacting stimulus alone at each of the 5 positions. Preferred or nonpreferred figure was positioned in the display's center, whereas the interacting shape was displayed at 1 of 5 positions (see Fig. 1B). Position of each of the 5 triplets of bars corresponds to the visual field position of the interacting shape, e.g., the bars in the top right corner correspond to the responses when the interacting stimulus was positioned in the upper contralateral field. Left and middle open bars of each triplet indicates the response to the preferred and nonpreferred figure presented alone at the display's center (see $A$ ). Mean responses to the combination of the preferred (nonpreferred) figure and interacting stimulus are indicated by the filled (hatched/shaded) bars. Response to the figure alone is indicated by arrows in the case of a larger response to the combined shapes than to the figure alone. Rightmost bar of each triplet shows the mean response to the interacting stimulus alone (shaded bar). Stippled horizontal line at each of the 5 positions corresponds to a net response of 0 spikes/sec, vertical lines indicate standard errors, and a calibration bar is shown at the central position. hemifield had a significant effect on the normalized responses to the combined stimulus $[F(1,85)=6.69 ; P<0.011 ; n=86]$. Normalized responses to combinations with an interacting stimulus in the ipsilateral part of the RF were stronger (median: 0.85; quartiles: $0.68-1.1$ ) than those with an identical stimulus in the contralateral part of the RF (median: 0.76; quartiles: 0.46-0.96).

Responses to the single preferred figure at a given position can be used as an estimate of the sensitivity of the neuron at that position. If differences in stimulus interaction between the two hemifields simply reflect RF sensitivity, then there should be a negative correlation between the normalized responses to the figure alone and the combination of figure and interacting stimulus. However, as shown in Fig. 5, the normalized responses to the preferred figure alone and to the shape combination were only weakly and positively correlated in both contralateral $(r=0.48 ; P<0.01 ; n=86)$ and ipsilateral visual field $(r=0.30 ; P<0.01 ; n=86)$. Moreover these correlations became nonsignificant after one and three outliers were rejected, respectively, in the contra- and ipsilateral data $(-$ in Fig. 5; contra: $r=0.12$, ns, $n=85$; ipsi: $r=0.13$, ns, $n=83$ ). Thus at the population level, the stronger shape interactions present in the contralateral visual field reflect a genuine difference in the degree of shape interaction within the two hemifields.

The upper and lower contralateral positions tended to be similar with regard to the suppressive effects elicited by the interacting stimulus: there was a significant difference in the suppression elicited at these two positions in only six neurons $(14 \%)$. It should be noted that the vertical asymmetry in the degree of suppression in these six neurons did not reflect RF sensitivity at these positions because the response to the preferred figure alone did not differ significantly between these positions for five of the six neurons.

For eight cells, we tested the influence of an interacting stimulus on the response to the central preferred figure when the interacting stimulus was placed at several eccentricities. Usually two, but up to a maximum of five eccentricities, spaced by at least $2^{\circ}$ and ranging from 1.8 and $10.8^{\circ}$, were tested. Figure 6 gives an example of a cell tested with the same interacting stimulus at four different eccentricities. The effect of the distance was significant [ANOVA; $F(3,138)=116.16$; $P<0.001]$. Note that figure and interacting stimulus overlapped only at $1.8^{\circ}$ eccentricity and that the preferred figure evoked a significant response when presented alone at all positions tested. This example shows that the response gradually decreased as the interacting stimulus moved closer to the display's center. Overall the radial distance of the interacting stimulus showed a significant main effect in five cells $(5 / 8$; $62 \%$ ), with four cells responding significantly less when the interacting stimulus was closer to the center (the 5th cell responded stronger when the interacting stimulus was closer to the center).

The lack of correlation between responses to single figures 
A
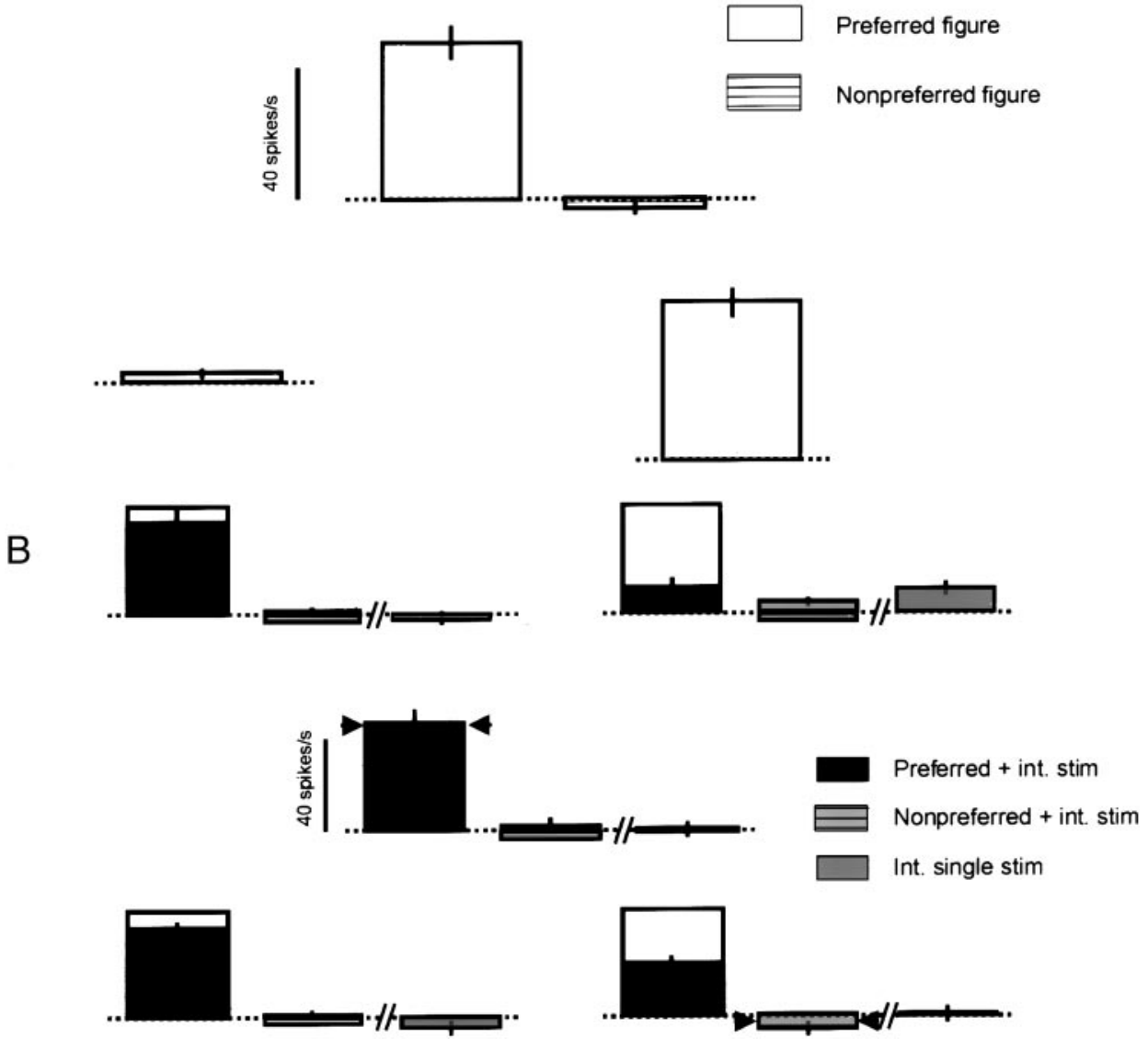

FIG. 4. Responses of an IT neuron showing suppression with the combined shapes only when the 2 shapes are nonoverlapping. Same conventions as Fig. 3.

and to combined shape configurations (Fig. 5) shows that effect of the position of the interacting shape is not due to differences in RF sensitivity. This suggests that the shape interactions are strongly nonlinear as has been reported in previous studies (Miller et al. 1993; Sato 1988, 1989, 1995). Indeed the responses to the combination of figure plus interacting stimulus could not be predicted from the sum of the responses to the constituent stimuli. The sum of the normalized responses to the interacting stimulus alone and preferred figure alone was not correlated with the normalized response to the combined stimulus $[r$ smaller than 0.30 (ns) in all eccentric positions, except for the ipsilateral upper position $(r=0.37 ; P=0.015)]$. Moreover the size of the suppression induced by an interacting stimulus did not depend on the amount of inhibition that this stimulus elicited when presented alone.

INFLUENCE OF THE SHAPE OF THE NONOVERLAPPING INTERACTING STIMULUS. The influence of the shape of the interacting stimulus was tested in 10 cells using at least two different shapes as the interacting stimulus. A two-way ANOVA was performed on the normalized responses of each cell with the shape of the interacting stimulus and eccentric position as factors. Figure 7 exemplifies a cell in which the shape of the interacting stimulus produced a significant main effect [ANOVA; $F(1,72)=52.58 ; P<0.001]$. Interacting stimulus $I$ reduced the response to the preferred figure in the center and at the contralateral lower position. Interacting stimulus II increased the response to the figure when it was displayed at eccentric positions, even if the response of the cell to the interacting stimulus II alone was not larger than that to the interacting stimulus I. Overall, shape of the interacting stimulus showed a significant main effect in four cells, whereas two neurons showed a significant interaction between shape and position (in 1 of these, the main effect of shape was also significant). Thus for $50 \%$ of the neurons tested, the shape of the interacting stimulus influenced the interaction between the two shapes. This may well be an underestimation since usually only two different shapes were tested.

INFLUENCE OF AN INTERACTING STIMULUS ON SHAPE SELECTIVITY AND PREFERENCE. Because the effect of the interacting stimulus was assessed for two different shapes that were selected based on the initial testing with 30 shapes, we could determine whether the presence of the interacting stimulus 
A

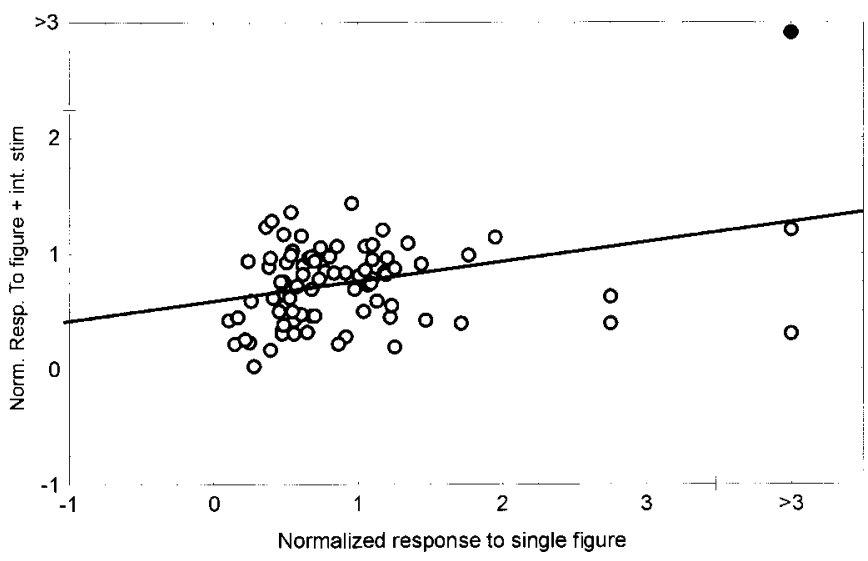

B

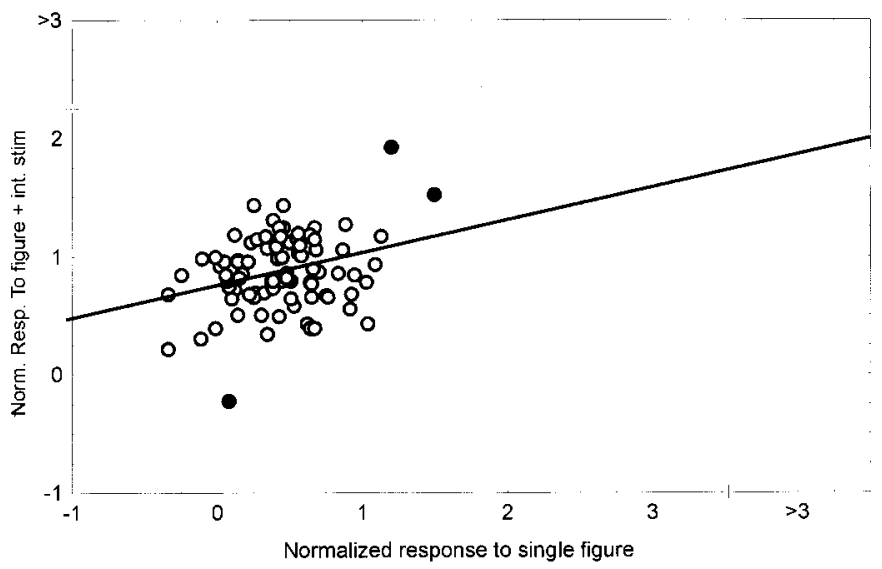

FIG. 5. Correlation of the normalized response to the combination of preferred shape and interacting stimulus and the normalized response to preferred shape presented alone at the same peripheral position as the interacting stimulus. Responses were normalized with respect to the response to the preferred figure alone at the display's center. $A$ : contralaterally positioned interacting stimulus and single figure. $B$ : ipsilaterally positioned interacting stimulus and single figure. Data of top and bottom positions are pooled for each hemifield $(n=86)$. When the filled data points are treated as outliers, the positive correlations became not significant.

influenced the shape selectivity and preference of the neurons. Figure 8 shows the distribution of the SIs (see METHODS) for single figures and for the different shape combinations. The median SIs were significantly smaller for each of the combined shape conditions than for the single figure (Wilcoxon matched pair test, $P<0.05$ ), although this effect was small when the interacting stimulus was located ipsilaterally. For each of the combined shape conditions, the SI was on average significantly $>0$ ( $t$-test; $P<0.05)$, indicating that although the degree of selectivity is reduced, the population of neurons still could discriminate the two shapes when these were combined with the interacting stimulus. Furthermore it should be noted that if all positions were considered together, only one neuron showed a significantly reversed shape preference $($ SI $<0)$ when shape combinations were tested.

\section{Absolute and relative position test}

In the second test, we have disentangled the absolute and relative positions of the interacting stimulus. Seventeen cells were tested at an eccentricity of $5.5^{\circ}$, and these results are summarized in Fig. 9. It is shown that the normalized response was reduced much more strongly for the nonoverlapping conditions when the interacting stimulus is presented at the center of the display (condition 4) than when the preferred figure is in that position (condition 3). The marked effect of reversing the positions of the two shapes is not simply due to the lower sensitivity for the peripheral stimulus because a change in the position of the preferred figure alone had much less effect (conditions 1 and 2). In the conditions where stimuli were overlapping (conditions 5 and 6 ), the average response was approximately the same whether both shapes were presented centrally or peripherally. It is worth noting that the sharpest decrease was observed in condition 4 , in which the interacting stimulus was presented centrally and the figure peripherally, a condition not tested previously.

A two-way ANOVA with "figure position" and "combination of shapes" (without overlap) as factors was done for each neuron, where the response to the combined stimulus was compared with the sum of the responses to the preferred and interacting stimuli alone at the other position. For 16 (94\%) of the neurons, the response to the combined shapes was less than the sum of the responses to the single figures (significant main effect of combination of shapes factor). Ten (59\%) cells showed a significant interaction between the two factors. Eight

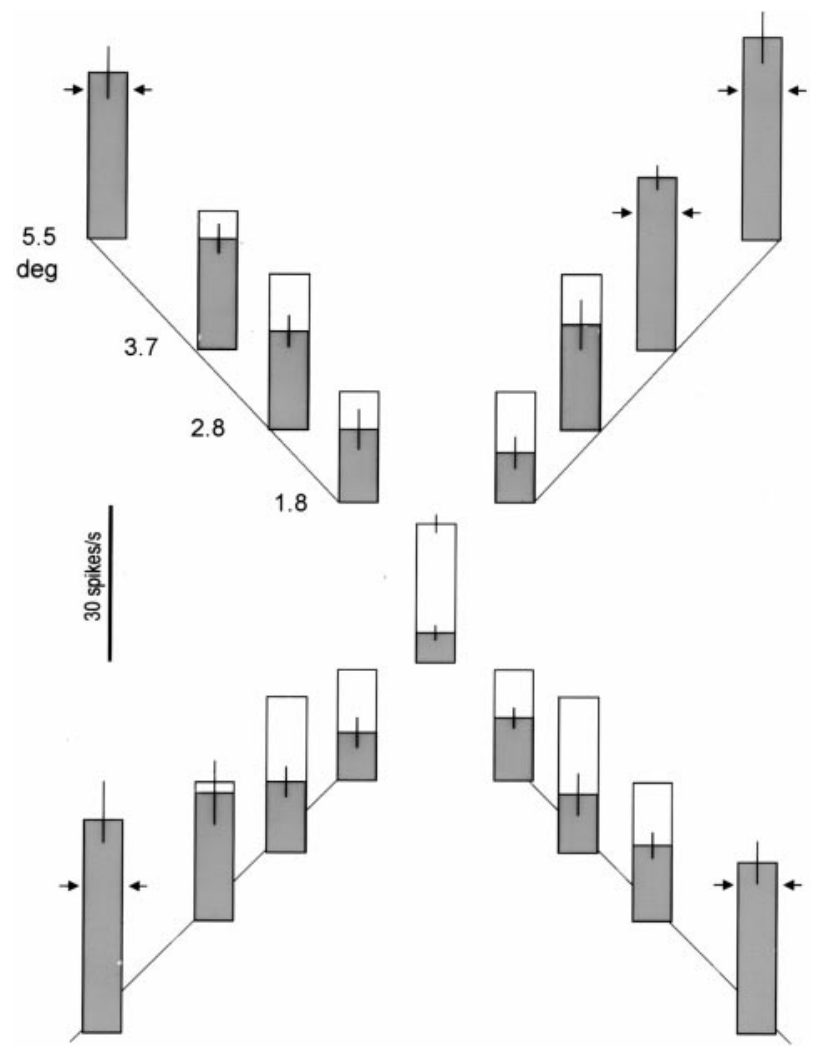

FIG. 6. Effect of the eccentricity of the interacting stimulus: example of a neuron tested with the interacting stimulus at 5 different eccentricities $(0-$ $\left.5.5^{\circ}\right) . \square, \rightarrow$, and $\leftarrow$, responses to the preferred figure alone in the center. $\square$, responses to the different combinations of the centrally presented preferred figure and the interacting stimulus. Position of the bars correspond to the visual field position of the interacting stimulus. Eccentricity of the interacting stimulus is indicated on the top left oblique axis using an ordinate scale. Preferred figure on its own evoked significant responses at all positions shown. Vertical bars indicate SEs. 

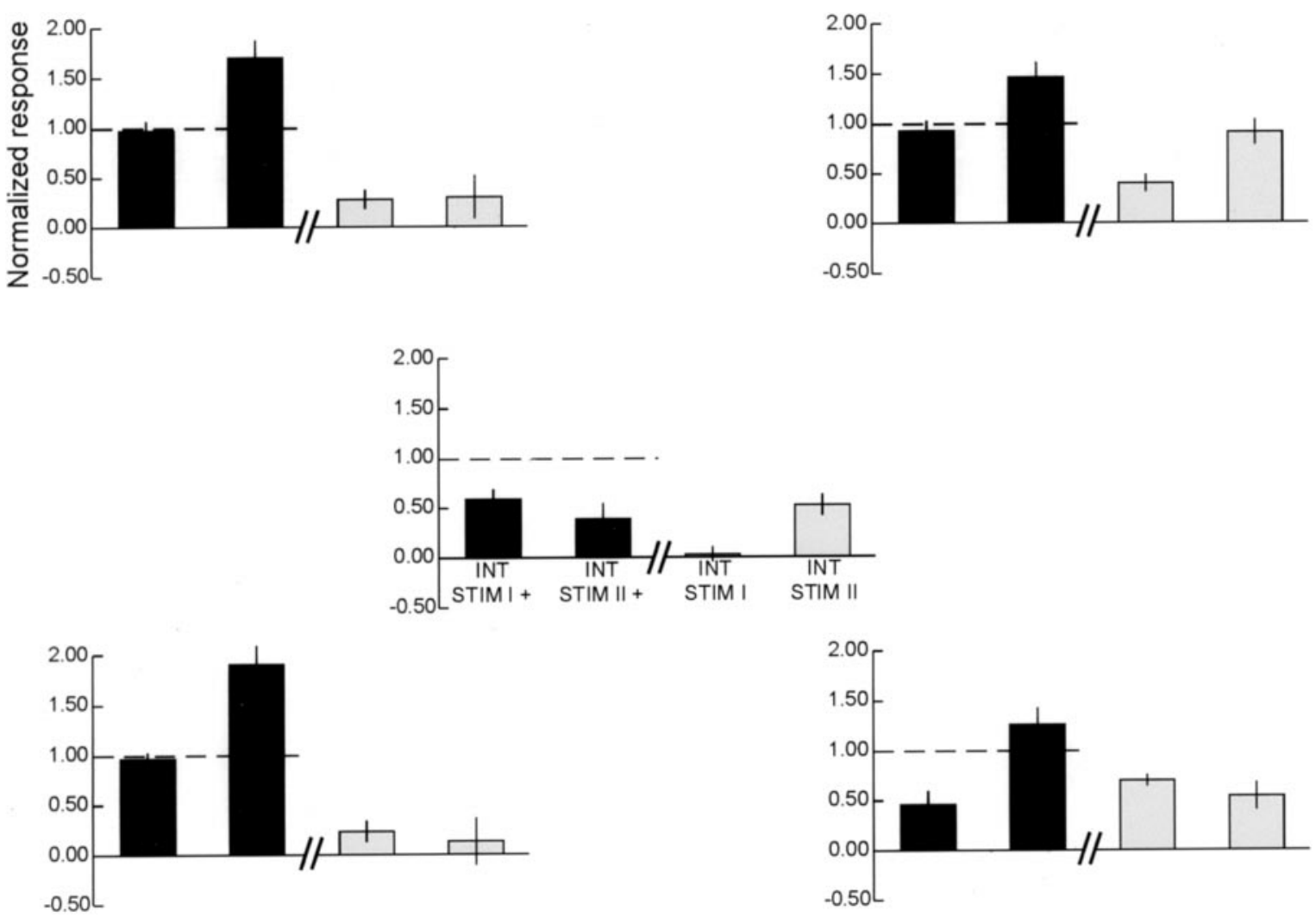

FIG. 7. Effect of the shape of the interacting stimulus. Normalized response to combinations of preferred figure and interacting stimuli for each of the 5 different positions of the interacting stimuli. Responses were normalized with respect to the response to

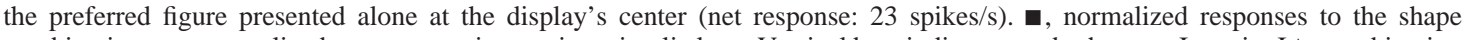
combinations; $\square$, normalized responses to interacting stimuli alone. Vertical bars indicate standard errors. Int. stim I+, combination of interacting shape I and preferred shape; Int. stim II +, combination of interacting shape II and preferred shape; Int. stim I, single presentation of interacting shape I. Int. stim II, single presentation of interacting shape II.

cells $(8 / 10)$ were suppressed more strongly when the interacting stimulus was in the center of the RF and the preferred figure was located in the periphery than in the opposite configuration, as was also apparent in the averaged data (Fig. 9). The other two cells showed the converse interaction. This analysis shows that in the majority of IT neurons the response is more sharply reduced when the interacting stimulus is in the center of the visual field.

In a second analysis, we performed a two-way ANOVA on conditions 3-6 with preferred figure position and overlap as factors. The main effect of overlap was significant for 9 neurons, and 12 neurons showed a significant interaction between the position and overlap factors. Two neurons responded significantly less in the overlap compared with the nonoverlap conditions, irrespective of the preferred figure's position (main effect of overlap without interaction). Responses were higher, or lower, in overlapping conditions compared with the nonoverlapping conditions (significant effect of the factor overlap) in three and four neurons, respectively, but the size of the overlap effect depended on the position of the preferred figure (significant interaction). In one neuron, the interaction was combined with a main effect of the factor position of the preferred figure. For seven neurons $(41 \%)$, the shape interactions were similar in overlap and nonoverlap conditions, when effects of absolute stimulus position were taken into account. In four of these neurons, the effect of overlap reversed depending on which of the two shapes occupied the center position with none of the main effects being significant. Typically the response was suppressed more strongly in the overlap than in the nonoverlap condition when the preferred figure was at the center, whereas the opposite was the case when the preferred figure was in the peripheral position. Thus the response of these neurons probably was determined by the absolute position of the interacting stimulus in the visual field, with the response strongly reduced when the interacting stimulus was presented centrally, whether or not there was any overlap with the figure.

Finally, as in the position test, the SIs were significantly lower in each of the combined compared with the single shape presentations, and, in a few neurons, the SIs became negative in the combined-shape conditions. However, none of these reversals in shape preference proved to be statistically significant.

\section{I S C U S S I O N}

When a preferred and nonpreferred shape are presented simultaneously in the receptive field of an IT neuron, the response to this combination is generally lower than to the preferred shape presented in isolation. This decrease can occur irrespective of whether the two shapes overlap and depends on the absolute and relative positions of the two shapes in the receptive field of the neuron. When the preferred figure was presented in the center of the display, shape interactions were 
A

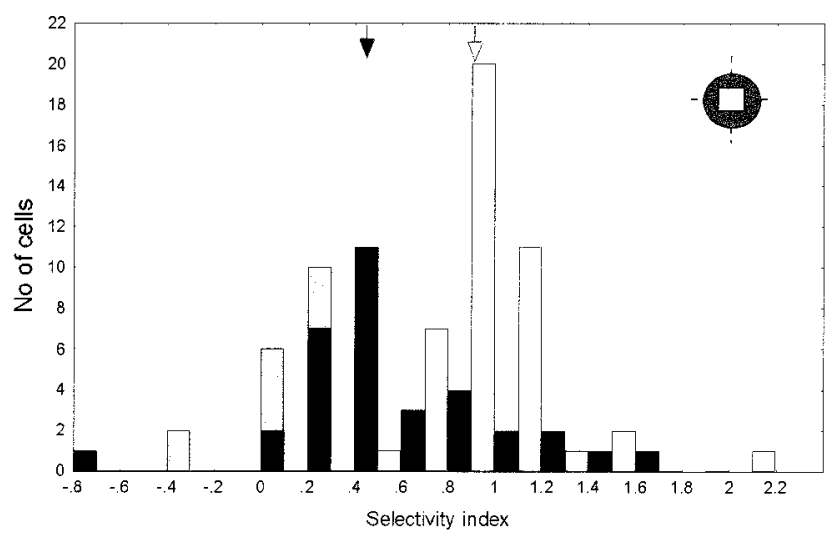

B

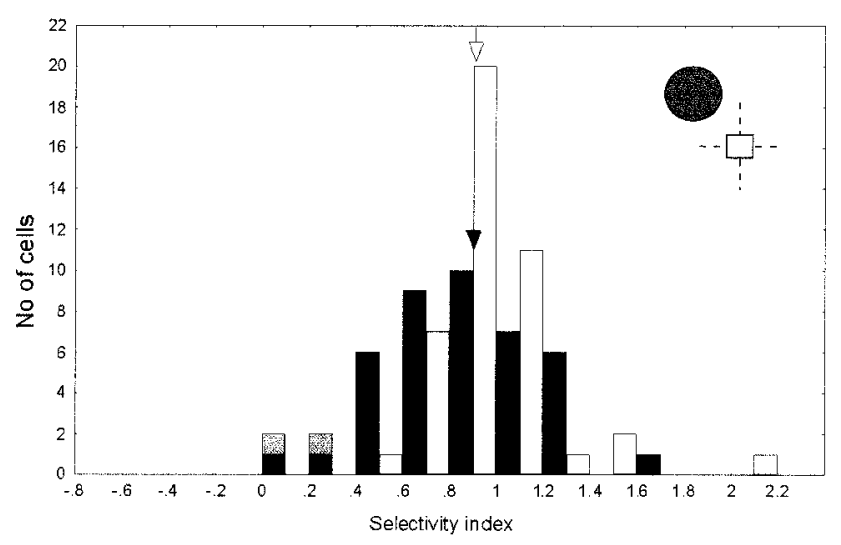

C

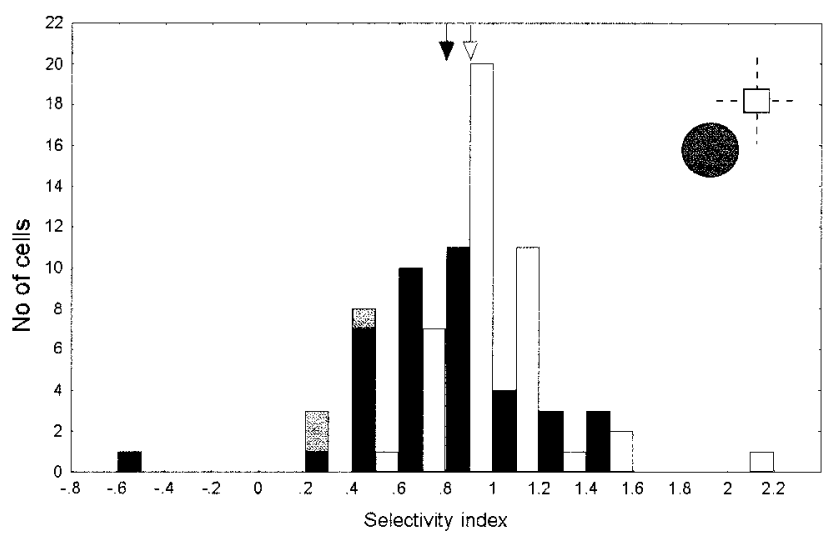

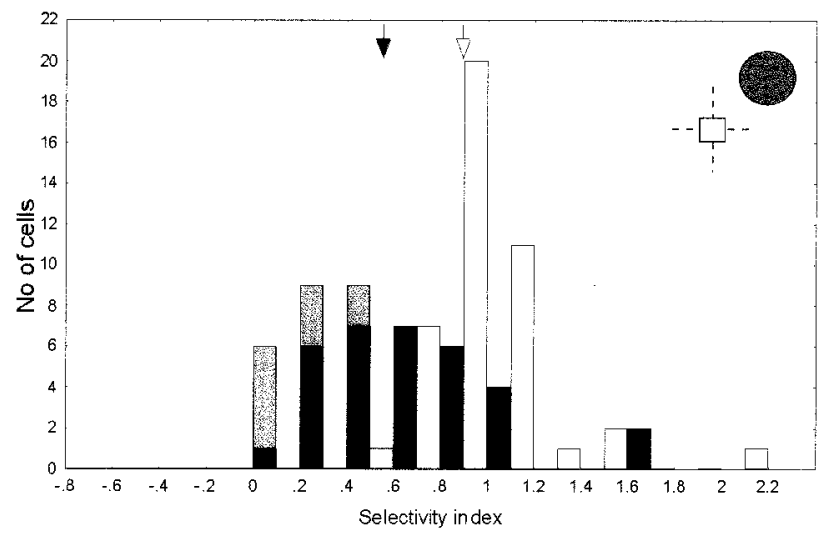

$\mathrm{E}$

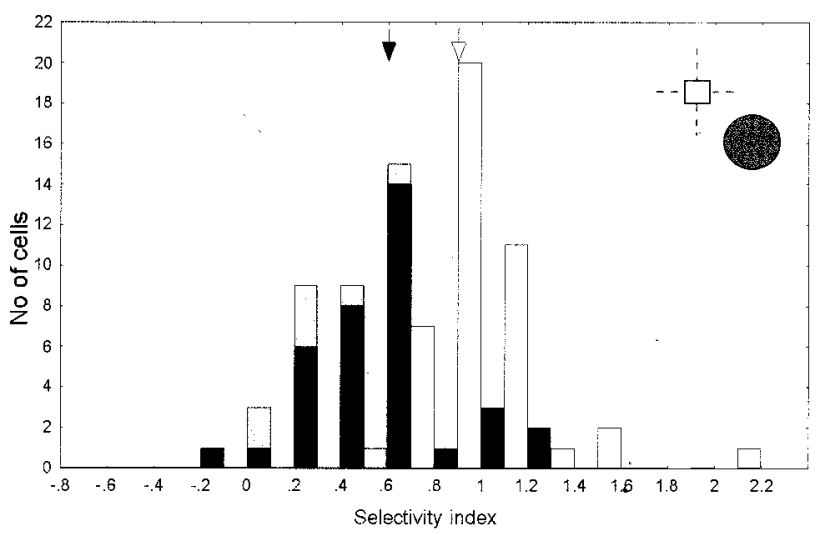

Single

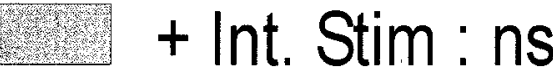

+ Int. Stim : sign

FIG. 8. Distribution of the selectivity index for the different positions of the interacting stimulus. Distribution of the selectivity index for single figures presented at the display's center are shown by open bars and for the different shape combinations by $\square$ and $\mathbf{\square}(n=43)$. a, those neurons for which responses to a combination with a preferred shape and one with a nonpreferred figure differed significantly. Inset: stimulus configuration ( $\square$ : figure; $\bullet$ : interacting stimulus). A: figure and interacting stimulus overlapped at the center of the display. $B$ and $C$ : interacting stimulus located ipsilaterally. $D$ and $E$ : interacting stimulus located contralaterally. $\quad \frac{1}{b}$, median of the selectivity index for single figures; $\downarrow$, median of the selectivity index for shape combinations.

stronger when the interacting stimulus was located in the contralateral as opposed to the ipsilateral hemifield and, on average, decreased with the eccentricity of the interacting stimulus. The effect of the position of this interacting shape on the degree of suppression was not correlated with the RF sensitivity profile for single shapes, indicating strong nonlinear interactions. The response was, on average, smallest when the interacting stimulus, rather than the preferred figure, was pre- 
A

\section{Single}

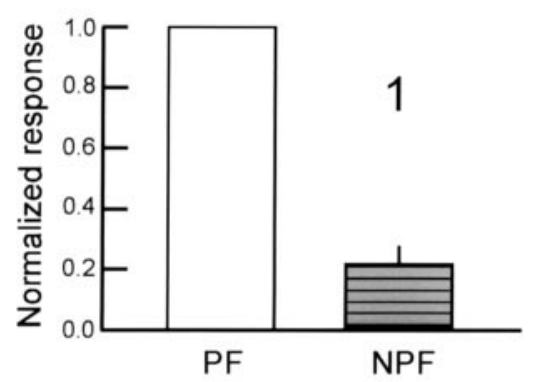

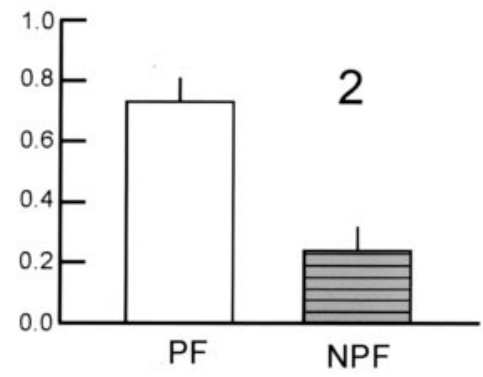

B

Non-overlapping
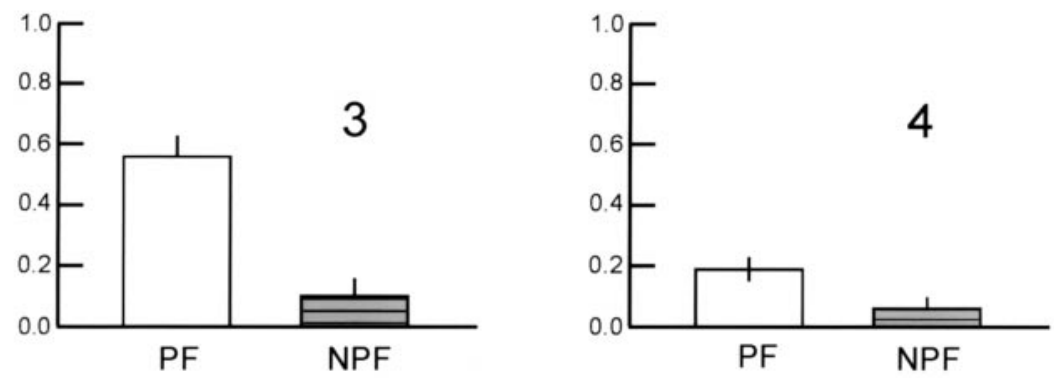

C

\section{Overlapping}
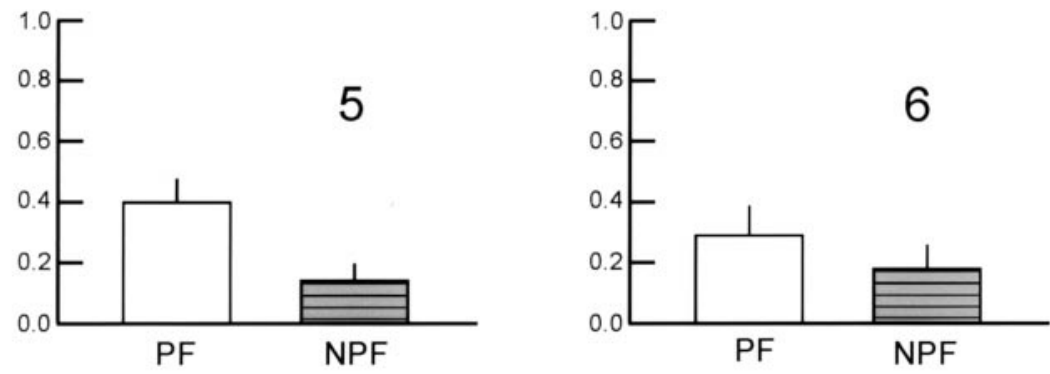

FIG. 9. Absolute and relative position test: population responses $(n=17)$. Responses were normalized with respect to response to the single preferred shape at the display's center $(n=17)$. A: figure presented alone at the display's center (1) or in the periphery (2). $B$ : nonoverlapping shape combinations. 3: figure at the center; 4: figure in the periphery; $C$ : overlapping shape combinations at the display's center (5) or in the periphery (6). D: interacting shape presented alone at the display's center (7) or in the periphery (8). Condition numbers correspond to those of Fig. 2. Open bars, preferred figure (PF); hatched/shaded bars, nonpreferred figure (NPF); shaded bars, interacting shape. Vertical bars indicate SEs.

D

\section{2nd stimulus}

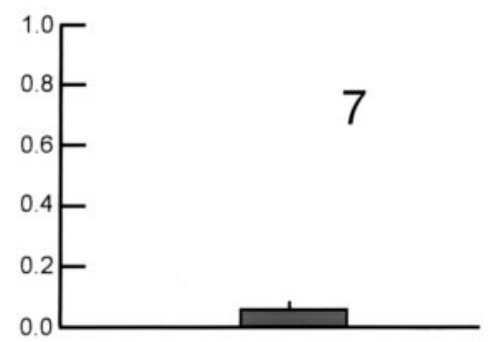

Center

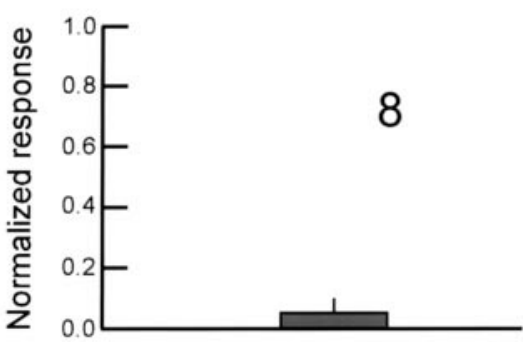

Periphery sented at the fixation position. In some neurons, the degree of interaction between two nonoverlapping shapes depended on the shape of the second stimulus. These results indicate that stimulus interactions within the receptive fields of IT neurons can be position and shape sensitive. Finally, we showed that the response to a combination of two nonpreferred shapes was usually lower than the response to a combination of the pre- ferred and a nonpreferred shape, indicating that the differential response to a pair of stimuli, presented in isolation, is maintained despite the presence of an additional stimulus.

\section{Shape interactions for overlapping versus nonoverlapping shapes}

In agreement with Missal et al. (1997), we found that when a preferred shape partially overlaps a nonpreferred shape, the 
response to this stimulus combination is, in most cells, lower than to the preferred shape presented alone. The finding of response suppression in nonoverlap conditions agrees with previous studies that either employed two peripherally presented shapes (Miller et al. 1993), used a sequential presentation of two simple stimuli in a discrimination task (Sato 1988) or tested face cells (Rolls and Tovee 1995).

In the position test, we found that response reduction in the overlap condition did not correlate with that in the nonoverlap conditions. However, in that test, the difference between the degree of suppression in the overlap and nonoverlap conditions could be due to either the overlap factor or to differences in the position of the interacting shape in the two conditions. By deconfounding these two factors in the absolute and relative position test, we found that the overlap factor did not affect the suppression in $\sim 40 \%$ of the neurons. This suggests that, for at least these neurons, the response reduction in the overlap condition reflects genuine shape interactions. Factors such as a "weak segmentation" of figure and second shape, or a response to the figure-second shape combination as a whole in the overlap conditions, may contribute to the response reduction in neurons in which the response differed between overlap and nonoverlap conditions.

\section{Position dependence of shape interactions}

We found that, within a single hemifield, the angular position of the interacting stimulus influenced the degree of suppression in only $14 \%$ of the neurons. In addition, we observed that the average response was lower when the interacting stimulus was placed contralaterally, rather than ipsilaterally, with respect to the central shape. Neither of the effects of the absolute position of the interacting stimulus are due to differences in the RF sensitivity profile as assessed with single shapes.

Rocha-Miranda et al. (1975) showed that the responses to stimuli in the ipsilateral part of the RF critically depend on the integrity of the forebrain commissures. Thus the weaker interactions observed for interacting stimuli placed in ipsilateral positions suggests that either this commissural input carries weaker inhibitory influences than the connections within a hemisphere or that the intrahemispheric connections between neurons preferring different shapes are more widespread and/or stronger than interhemispheric connections.

Sato (1988), using different stimulus and task conditions than those of the present study, also found less suppression when the second stimulus was placed in the ipsi- compared with the contralateral hemifield. However, Rolls and Tovee (1995) reported no hemifield-dependent effects in the degree of interaction of two faces in a task very similar to the present study. The results of their study and ours combined indicate that the contribution of the ipsilateral input to these stimulus interactions depends either on the complexity of the stimulus (faces versus shapes) or, more likely, on the degree of stimulus complexity represented by the neuron (face vs. shape selective neurons).

\section{Comparison with stimulus interactions in other visual areas}

Stimulus interactions have been documented in several visual areas such as areas V1, MT/V5, and V4. In most of these studies, the second stimulus was placed well outside the "classical" RF but it should be noted that interactions in V1
(Knierim and Van Essen 1992; Orban et al.1989; Sillito et al. 1995), MT/V5 (Allman et al. 1985; Raiguel et al. 1995; Tanaka et al. 1986; Xiao et al. 1997), and V4 (Desimone and Schein 1987) also can occur when both stimuli are positioned inside the RF. Miller et al. (1993) suggested that IT responses should be most suppressed when the second stimulus is located outside the RF. However, we found at best a weak correlation between the response to a single shape at a particular visual field position and the degree of suppression by an interacting shape at that position (Fig. 5). Furthermore when eccentricity did have an effect, it more frequently entailed a reduction in the degree of interaction with increasing eccentricity than it did the opposite. This is contrary to what one might expect if the suppressive effects increase when the interacting stimulus is positioned closer to the borders of, or outside, the RF.

In areas V1, MT/V5, and V4, stimulus interactions are highly specific for the stimulus properties of the second stimulus, e.g., its orientation, color, or direction of motion. In contrast to these observations, Miller et al. (1993) found that the degree of suppression in IT neurons was similar regardless of whether or not identical shapes were paired in the periphery, whereas Rolls and Tovee (1995) found that the degree of suppression depended on whether or not the second face was preferred by the neuron when presented alone. We observed that in 50\% of the somewhat limited number of neurons tested, the shape interactions depended significantly on the shape of the interacting stimulus, suggesting that stimulus interactions in IT depend on the properties of the interacting stimulus.

\section{Suppression and attention}

The average response of the shape selective neurons was lowest when the nonpreferred interacting stimulus was at the fixation position and the preferred figure at the peripheral position. This finding, extending a similar observation made by Rolls and Tovee (1995) in testing face cells, suggests that, in general, stimuli at the fovea are given more weight in the stimulus interaction process than those at peripheral positions. It is possible that the central stimulus had a greater effect in these fixating animals because attention presumably was directed toward that position. However, it should be noted that the monkey was not required to attend any of the stimuli and thus that the greater weight given to stimuli positioned at the fovea may reflect the default operation of the system. It also should be noted that in anesthetized monkeys, responses in IT neurons are also usually stronger at the fovea than at more peripheral positions (Desimone and Gross 1979; Gross et al. 1972), suggesting that in neutral attentional states foveally presented stimuli are indeed more effective than peripheral stimuli. Of course, attention to a particular stimulus could increase its weight in the stimulus interaction process and thus affect the default stimulus interactions. Indeed, it has been reported that the responses of IT neurons to a stimulus can depend on whether or not that stimulus is being attended (Chelazzi et al. 1993; Moran and Desimone 1985). In the present experiments, the locus of attention coincided with the center of gaze, which is the most common situation in natural vision, and thus we feel that this study provides a valid picture of stimulus interactions that occur in IT during natural vision. 


\section{Relevance of stimulus interactions for object coding}

The present results, as well as those of Missal et al. (1997), suggest that stimulus interactions mainly reduce the response of the neuron without affecting the neuron's stimulus preference. As a consequence, those neurons preferring either stimulus of the combination still will be more active than neurons representing other stimuli and thus will be able to signal the presence of their preferred shapes despite the simultaneous presence of other, interacting stimuli. The invariance of the shape preference thus simplifies the read-out of the activity profile of the population of neurons.

Because these stimulus interactions depend, in a significant number of neurons, on the position and shape of the two stimuli, these neurons will be sensitive to the stimulus configuration and thus these interactions can contribute to the coding of the relationships between object components (Missal et al. 1997). This may have far-reaching consequences for object coding by IT neurons. If objects are represented by several groups of neurons wherein each group, because of its selectivity for a "critical feature", (Tanaka 1996), signals the presence of an object component, one needs a mechanism to signify that various components belong together. Because of their shape and position selectivity, suppressive shape interactions could contribute to binding parts together. Indeed this suppression may be released only when appropriate shapes are present at appropriate positions with respect to the neuron's preferred component (critical feature). Whether or not shape interactions play this important role in object coding needs further work.

We thank M. De Paep, G. Vanparrys, P. Kayenbergh, and G. Meulemans for technical assistance, W. Spileers for surgical assistance, L. Arckens for histological assistance, and S. Raiguel for critical reading of the manuscript.

This work was supported by Fonds voor Wetenschappelijk Onderzoek (FWO)-Vlaanderen Grant G. 0172.96, Interuniversitaire Attractie Pool P4/22, Geconcerteerde Onderzoeksactie 95-99/6, Geneeskundige Stichting Koningin Elizabeth. C.-Y. Li held a senior fellowship of the Research Council of the Katholieke Universitet Leuven and was on leave from the Shangai Institute of Physiology, Chinese Academy of Sciences, Shangai, China. M. Missal was on leave from and partially supported by the Laboratory of Neurophysiology, Université Catholique de Louvain, Woluwe, Belgium. R. Vogels is a research associate at FWO-Vlaanderen.

Address for reprint requests: R. Vogels, Laboratorium voor Neuro- en Psychofysiologie, Faculteit Geneeskunde, Katholieke Universitet Leuven, Campus Gasthuisberg, Herestraat 49, B-3000 Leuven, Belgium.

Received 10 November 1998; accepted in final form 4 February 1999.

\section{REFERENCES}

Allman, J., Miezin, F., And McGuiness, E. Stimulus specific responses from beyond the classical receptive field. Annu. Rev. Neurosci. 8: 407-430, 1985.

Chelazzi, L., Miller, E. K., Duncan, J., And Desimone, R. A neural basis of visual search in inferior temporal cortex. Nature 363: 345-347, 1993.

DEAN, P. Effects of inferotemporal lesions on the behavior of monkeys. Psychol. Bull. 83: 41-71, 1976.

Desimone, R., Albright, T. D., Gross, C. G., and Bruce, C. Stimulusselective properties of inferior temporal neurons in the macaque. J. Neurosci. 4: 2051-2062, 1984.

Desimone, R. and Gross, C. G. Visual areas in the temporal cortex of the macaque. Brain Res. 178: 363-380, 1979.
Desimone, R. And Schein, S. J. Visual properties of neurons in area V4 of the macaque: sensitivity to stimulus form. J. Neurophysiol. 57: 835-868, 1987.

Gross, C. G., Rocha-Miranda, C. E., And Bender, D. B. Visual properties of neurons in inferotemporal cortex of the macaque. J. Neurophysiol. 35: 96-111, 1972.

Judge, S. J., Richmond, B. J., And CHu, F. C. Implantation of magnetic search coils for measurement of eye position: an improved method. Vision Res. 20: 535-538, 1980

KIRK, R. E. Experimental Design: Procedure for the Behavioral Sciences. Belmont, CA: Brooks-Cole, 1968.

KNIERIM, J. J. AND VAN EsSEN D. C. Neuronal responses to static textures patterns in area V1 of the alert macaque monkey. J. Neurophysiol. 67: 961-980, 1992.

Komatsu, H., Ideura, Y., KaJi, S., And Yamane, S. Color selectivity in the inferior temporal cortex of the awake macaque monkey. J. Neurosci. 12: 408-424, 1992.

Kovács, G., Vogels, R., AND OrBan, G. A. Selectivity of macaque inferior temporal neurons for partially occluded shapes. J. Neurosci. 15: 1984-1997, 1995.

Logothetis, N. K. And Sheinberg, D. L. Visual object recognition. Annu. Rev. Neurosci. 19: 577-621, 1996.

Miller, E. K., Gochin, P. M., And Gross, C. G. Suppression of visual responses of neurons in inferior temporal cortex of the awake macaque by addition of a second stimulus. Brain Res. 616: 25-29, 1993.

Missal, M., Vogels, R., And Orban, G. A. Interaction between shapes in the receptive field of neurons in inferior temporal cortex. Soc. Neurosci. Abstr. 22: 1616,1996

Missal, M., Vogels, R., AND Orban G. A. Responses of macaque inferior temporal neurons to overlapping shapes. Cereb. Cortex 7: 758-767, 1997.

Moran, J. And Desimone, R. Selective attention gates visual processing in the extrastriate cortex. Science 229: 782-784, 1985.

Orban, G. A., Lagae, L., Raiguel, S., Gulyas, B., and Maes, H. Analysis of complex motion signals in the brain of cats and monkey. In: Models of Brain Function, edited by R.M.J. Cotterill. Cambridge: Cambridge, 1989, p. $151-165$.

Raiguel, S., Van Hulle, M. M., Xiao, D. K., Marcar, V. L., and Orban, G. A. Shape and spatial distribution of receptive fields and antagonistic motion surrounds in the middle temporal area (V5) of the macaque. Eur. J. Neurosci. 7: 2064-2082, 1995.

Rocha-Miranda, C. E., Bender, D. B., Gross, C. G., and Mishkin, M. Visual activation of neurons in inferotemporal cortex depends on striate cortex and forebrain commissures. J. Neurophysiol. 38: 475-491, 1975.

Rolls, E. T. AND ToveE, M. J. The responses of single neurons in the temporal visual cortical areas of the macaque when more than one stimulus is present in the receptive field. Exp. Brain Res. 103: 409-420, 1995.

SATO, T. Effects of attention and stimulus interaction on visual responses in inferior temporal neurons in macaque. J. Neurophysiol. 60: 344-363, 1988.

SATO, T. Interactions of visual stimuli in the receptive fields of inferior temporal neurons in awake macaques. Exp. Brain Res. 77: 23-30, 1989.

SATO, T. Interactions between two different stimuli in the receptive fields of inferior temporal neurons in macaques during marching behavior. Exp. Brain Res. 105: 209-219, 1995.

Sillito, A. M., Grieve, K. L., Jones, H. E., Cudeiro, J., And Davis, J. Visual cortical mechanisms detecting focal orientation discontinuities. Nature 378: 492-496, 1995

Tanaka, K., Hikosaka, K., Saito, H., Yuka, M., Fukuda, Y., and Iwai, E. Analysis of local and wide-field movements in the superior temporal visual areas of the macaque monkey. J. Neurosci. 6: 134-144, 1986.

TAnaka, K., Saito, H., Fukada, Y., And Moriya, M. Coding visual images of objects in the inferiotemporal cortex of the macaque monkey. J. Neurophysiol. 66: 170-189, 1991.

TANAKA, K. Inferotemporal cortex and object vision. Annu. Rev. Neurosci. 19: 109-139, 1996.

Xiao, D. K., Raiguel, S., Marcar, V., and Orban, G. A. Spatial distribution of the antagonistic surround of MT/V5 neurons. Cereb. Cortex 7: 662-677, 1997. 